\title{
On three-body B decays to charm
}

\author{
P. Colangelo ${ }^{\mathrm{a}}$ \\ ${ }^{a}$ Istituto Nazionale di Fisica Nucleare, Sezione di Bari, \\ via Amendola n.173, I-70126 Bari, Italy
}

I briefly describe the use of the three-body decay modes $B^{0} \rightarrow D^{(*)-} D^{(*) 0} K^{+}$to investigate open issues in charmed meson spectroscopy, and of the time dependent $B^{0}\left(\overline{B^{0}}\right)(t) \rightarrow D^{-} D^{+} \pi^{0}$ transitions for a measurement of $\cos (2 \beta)$.

\section{1. $B^{0} \rightarrow D^{(*)-} D^{(*) 0} K^{+}$AND CHARMED MESON SPECTROSCOPY}

Important physical information can be obtained analyzing nonleptonic many-body $B$ decays, once clean channels have been recognized and selected. Remarkable examples are the threebody modes into pairs of charmed mesons and a light pseudoscalar meson, for which one can attempt a theoretical treatment based on heavy quark and chiral light meson symmetries [1]. A few of such modes have been recently observed. Here I consider

$$
\begin{aligned}
& B^{0} \rightarrow D^{*-} D^{(*) 0} K^{+} \\
& B^{0} \rightarrow D^{-} D^{(*) 0} K^{+}
\end{aligned}
$$

observed by the BABAR [2] and BELLE 3] collaborations with a (preliminary) measurement of the branching fractions. Such modes are useful for shedding light on open issues of the charmed meson spectroscopy; from the theoretical point of view, they can be used to assess, e.g., the validity of the factorization ansatz.

The main idea is that the decays (11) and (2) essentially proceed through two-body intermediate states, $B^{0} \rightarrow D^{(*)-} D_{s}^{X}$, followed by the strong transition $D_{s}^{X} \rightarrow D^{(*) 0} K^{+}$(fig.1), the intermediate $D_{s}^{X}$ being charmed strange mesons. In the factorization approach [4], the $B^{0} \rightarrow D^{(*)-} D_{s}^{X}$ amplitudes are expressed as the product of the semileptonic $B^{0} \rightarrow D^{(*)-}$ matrix element and the $D_{s}^{X}$ current-vacuum matrix element. When the infinite charm quark mass limit is exploited, the only contributions with non-vanishing $D_{s}^{X}$

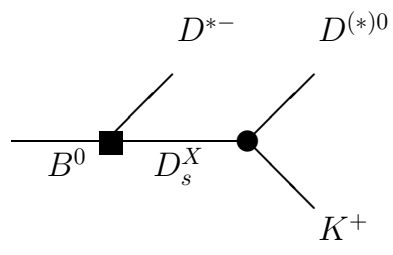

Figure 1. Polar diagram contributing to the modes $B^{0} \rightarrow D^{*-} D^{(*) 0} K^{+}$. The box represents a weak transition, the dot a strong vertex.

current-vacuum matrix elements correspond to the states $D_{s}^{X}=D_{s}^{*}$ and $D_{s 0}$ (with their radial excitations) for $B^{0} \rightarrow D^{*-} D^{0} K^{+}$, and $D_{s}^{X}=D_{s}^{*}$, $D_{s}$ and $D_{s 1}^{*}$ (together with their radial excitations) for $B^{0} \rightarrow D^{*-} D^{* 0} K^{+}$. Analogous selection rules hold for $B^{0} \rightarrow D^{-} D^{(*) 0} K^{+} . D_{s 0}$ and $D_{s 1}^{*}$ are charmed mesons with $J^{P}=0^{+}, 1^{+}$, respectively, belonging to the $s_{\ell}^{P}=\frac{1}{2}^{+}$heavy meson $(\bar{s} c)$ doublet, $s_{\ell}^{P}$ being the spin-parity of the light degrees of freedom in the meson. Such states are still unobserved, therefore it is worth identifying suitable channels for individually studying their features.

In order to calculate the amplitudes in fig.1] one needs the effective couplings parametrizing the vertices $D^{0} K^{+} D_{s}^{*}, D^{0} K^{+} D_{s 0}, D^{* 0} K^{+} D_{s}^{*}$, 
$D^{* 0} K^{+} D_{s}, D^{* 0} K^{+} D_{s 1}^{*+}$ (and analogous vertices involving radial $D_{s}^{X}$ resonances). In the heavy quark limit, all the couplings can be expressed in terms of two independent coupling constants, $g$ and $h$ for negative and positive parity $D_{s}^{X}$ states, respectively. This can be shown considering the effective QCD lagrangian, with heavy quark spinflavour and light quark chiral symmetries [5], that describes the interactions of heavy negative and positive parity mesons with the light pseudoscalar mesons:

$$
\begin{aligned}
\mathcal{L}_{I} & =i g \operatorname{Tr}\left\{H_{b} \gamma_{\mu} \gamma_{5} \mathcal{A}_{b a}^{\mu} \bar{H}_{a}\right\} \\
& +\left[i h \operatorname{Tr}\left\{H_{b} \gamma_{\mu} \gamma_{5} \mathcal{A}_{b a}^{\mu} \bar{S}_{a}\right\}+\text { h.c. }\right] .
\end{aligned}
$$

The fields $H_{a}$ in (3) describe the $J^{P}=\left(0^{-}, 1^{-}\right)$ $\bar{q} Q$ mesons with $s_{\ell}^{P}=\frac{1}{2}^{-}$:

$H_{a}=\frac{(1+\gamma)}{2}\left[P_{a \mu}^{*} \gamma^{\mu}-P_{a} \gamma_{5}\right]$,

the operators $P_{a}^{* \mu}$ and $P_{a}$ respectively annihilating the $1^{-}$and $0^{-}$mesons of four-velocity $v$ ( $a=u, d, s$ is a light flavour index). Analogously, the fields $S_{a}$ describe positive parity $s_{\ell}^{P}=\frac{1}{2}^{+}$ states:

$S_{a}=\frac{(1+\not)}{2}\left[P_{a \mu}^{\prime *} \gamma^{\mu} \gamma_{5}-P_{a}^{\prime}\right]$.

The octet of the light pseudoscalar mesons is included in (3) through the field $\xi=\exp \left(\frac{i \mathcal{M}}{f_{\pi}}\right)$, with $f_{\pi}=131 \mathrm{MeV}$ and $\mathcal{M}$ given by

$$
\left(\begin{array}{ccc}
\sqrt{\frac{1}{2}} \pi^{0}+\sqrt{\frac{1}{6}} \eta & \pi^{+} & K^{+} \\
\pi^{-} & -\sqrt{\frac{1}{2}} \pi^{0}+\sqrt{\frac{1}{6} \eta} & K^{0} \\
K^{-} & \bar{K}^{0} & -\sqrt{\frac{2}{3}} \eta
\end{array}\right)
$$

Moreover, $\mathcal{A}_{\mu b a}=\frac{1}{2}\left(\xi^{\dagger} \partial_{\mu} \xi-\xi \partial_{\mu} \xi^{\dagger}\right)_{b a}$.

It is straightforward to derive the relations of $g_{D_{s}^{*} D K}, g_{D_{s}^{*} D^{*} K}$ and $g_{D^{*} D_{s} K}$ to the effective coupling $g$, and of $g_{D_{s 0} D K}$ and $g_{D_{s 1}^{*} D^{*} K}$ to $h$, using (3)-(6). The full widths of the intermediate states can also be expressed in terms of $g$ and $h$, in the approximation of dominance of two-body decay modes. The masses of positive parity mesons can be taken from theoretical determinations: $m_{D_{s 0}} \simeq m_{D_{s 1}^{*}}=m_{D_{s}}+\Delta$, with $\Delta \simeq 0.5 \mathrm{GeV}[6]$.
The calculation of $B \rightarrow D^{(*)} D_{s}^{X}$, in the factorization approach, requires the semileptonic $B^{0} \rightarrow$ $D^{(*)-}$ matrix element and the $D_{s}^{X}$ decays constant. In the heavy quark limit, the former is given in terms of the Isgur-Wise function $\xi_{I W}$, while the decay constants $f_{D_{s}}$ and $f_{D_{s}^{*}}$, as well as $f_{D_{s 0}}$ and $f_{D_{s 1}^{*}}$, are simply related [7]. Other parameters, CKM elements and QCD coefficients, appear in the same combination as in the twobody $B^{0} \rightarrow D^{*-} D_{s}^{*}$ amplitude, the branching ratio of which is rather accurately known [8]; therefore, it is useful to express such combinations in terms of the $D^{*-} D_{s}^{*}$ branching fraction.

The contributions to the channels (1),(2) related to the radial excitations of negative and positive parity intermediate mesons can be estimated to represent less than $15 \%$ than the contribution of the corresponding low-lying states, using, e.g., the approach described in ref. [9].

The measurement of the ratios $\mathcal{B}\left(B^{0} \rightarrow\right.$ $\left.D^{*-} D^{0} K^{+}\right) / \mathcal{B}\left(B^{0} \rightarrow D^{*-} D_{s}^{*}\right)$ and $\mathcal{B}\left(B^{0} \rightarrow\right.$ $\left.D^{*-} D^{* 0} K^{+}\right) / \mathcal{B}\left(B^{0} \rightarrow D^{*-} D_{s}^{*}\right)$ [2,8] constrains the effective couplings $g$ and $h$. The experimental central values are obtained for $(g, h)=$ $(0.05,-0.59)$ and $(g, h)=(0.0,+0.60)$. As depicted in fig.2, the allowed regions in the plane $(g, h)$ are tightly bounded along the $h$ direction, in the range $|h|=0.6 \pm 0.2$ compatible with the expectations [6], while the dependence on $g$ is mild, the range extending over all the presently permitted values between $g=0$, the result $g=0.59 \pm 0.01 \pm 0.07$ obtained by the CLEO collaboration [10] and the upper bound $g<0.76$ from the ACCMOR collaboration 11, [12]. This means that the main contributions to the processes in (11) are not the $0^{-}$and $1^{-}, D_{s}$ and $D_{s}^{*}$ intermediate states, but the positive parity $0^{+}$ and $1^{+}$states $D_{s 0}$ and $D_{s 1}^{*}$, and therefore the $B^{0} \rightarrow D^{*-} D^{0} K^{+}$and $B^{0} \rightarrow D^{*-} D^{* 0} K^{+}$decay modes are well suited for separately studying the properties of the $D_{s 0}$ and $D_{s 1}^{*}$ resonances by the analysis of Dalitz plots that are expected as depicted in fig. 3. Broad resonances should be observed in the $D^{(*) 0} K^{+}$invariant mass, with $\Gamma\left(D_{s 0}\right) \simeq 180 \mathrm{MeV}$ and $\Gamma\left(D_{s 1}^{*}\right) \simeq 165 \mathrm{MeV}$. The same structures are expected in the modes $B^{0} \rightarrow D^{-} D^{0} K^{+}$and $B^{0} \rightarrow D^{-} D^{* 0} K^{+}$.

An interesting problem concerns the reduction 
of the two main uncertainties of the approach, i.e. the use of the heavy quark limit both for beauty and charm quarks, and the factorization ansatz employed in the nonleptonic matrix elements. From the theoretical point of view, a quantitative assessment of the role of such approximations is a nontrivial task and one has to analyze hints from the measurements. The decay modes (1,2) are different from the processes for which factorization has been proved in the infinite $b$ mass limit 13. Nevertheless, for channels of the class we are considering, i.e. color allowed $\mathrm{B}$ transitions to pairs of charmed mesons, the factorization model produces results in agreement with the available data, within the current experimental uncertainties [14]. The approach proposed here can be considered as a further test of factorization for three-body decays. The other main uncertainty is the use of the infinite mass limit for the charm quark. Finite charm quark mass effects can be at the origin of a deviation observed in the (preliminary) measurement of the branching fractions of (2) with respect to the results obtained in the infinite limit.

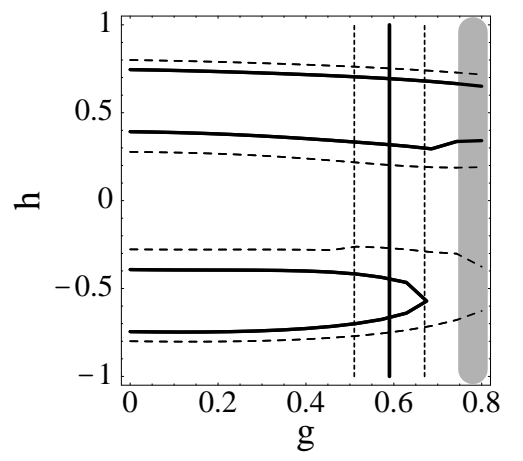

Figure 2. Allowed $1-\sigma$ (continuous lines) and 2$\sigma$ (dashed lines) regions in the $(g, h)$ plane. The vertical lines represent the result in ref.[10], the shaded area corresponds to the bound in ref. [11].
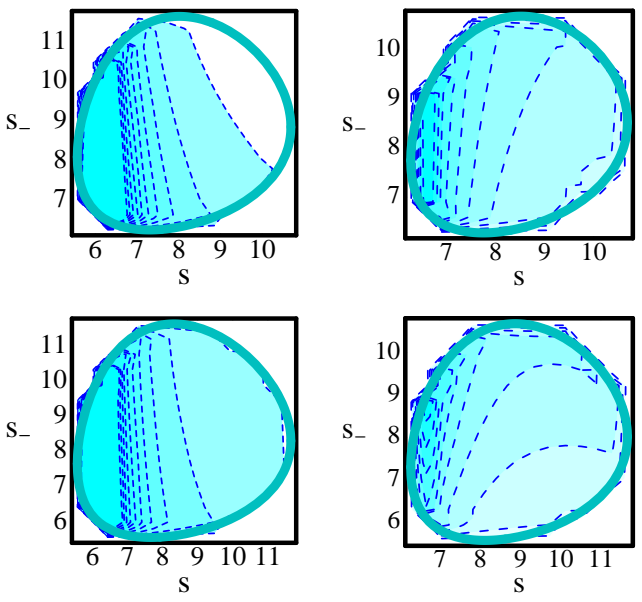

Figure 3. Dalitz plot of $B^{0} \rightarrow D^{*-} D^{0} K^{+}$(upleft), $D^{*-} D^{* 0} K^{+}$(up-right), $D^{-} D^{0} K^{+}$(downleft) and $D^{-} D^{* 0} K^{+}$(down-right). Units of $s=$ $\left(p_{D^{(*) 0}}+p_{K}\right)^{2}$ and $s_{-}=\left(p_{D^{(*)-}}+p_{K}\right)^{2}$ are $\mathrm{GeV}^{2}$.

\section{2. $B^{0}\left(\overline{B^{0}}\right)(t) \rightarrow D^{+} D^{-} \pi^{0}$ AND THE WEAK PHASE $\beta$}

At the B factories, the analysis of the timedependent Dalitz plot of the three-body modes $B^{0}\left(\overline{B^{0}}\right) \rightarrow D^{-} D^{+} \pi^{0}$ would be useful for the investigation of $\mathrm{CP}$ violating effects in neutral $B$ decays and, in particular, to get new information on the weak phase $\beta$ [15]. This can be easily understood, since the time-dependent decay probabilities of states identified at $t=0$ as $B^{0}$ and $\overline{B^{0}}$ respectively read:

$$
\begin{aligned}
\mid A\left(\left.B^{0}(t) \rightarrow D^{+} D^{-} \pi^{0}\right|^{2}\right. & \sim G_{0}\left(s_{+}, s_{-}\right) \\
+G_{c}\left(s_{+}, s_{-}\right) \cos (\Delta m t) & -G_{s}\left(s_{+}, s_{-}\right) \sin (\Delta m t), \\
\mid A\left(\left.\overline{B^{0}}(t) \rightarrow D^{+} D^{-} \pi^{0}\right|^{2}\right. & \sim G_{0}\left(s_{-}, s_{+}\right) \\
-G_{c}\left(s_{-}, s_{+}\right) \cos (\Delta m t) & \left.+G_{s}\left(s_{-}, s_{+}\right) \sin (\Delta m t)\right],
\end{aligned}
$$

with

$$
\begin{aligned}
G_{s}\left(s_{+}, s_{-}\right)= & -2 \sin (2 \beta) \operatorname{Re} \tilde{G}_{s}\left(s_{+}, s_{-}\right) \\
& +2 \cos (2 \beta) \operatorname{Im} \tilde{G}_{s}\left(s_{+}, s_{-}\right) .
\end{aligned}
$$

Therefore, through the analysis of $B^{0}\left(\overline{B^{0}}\right)(t) \rightarrow$ $D^{-} D^{+} \pi^{0}$ one envisages the possibility to access both $\sin (2 \beta)$ and $\cos (2 \beta)$, and therefore to solve 
the discrete ambiguity $\beta \rightarrow \frac{\pi}{2}-\beta$ still present in current measurements from, e.g., CP asymmetries in $B \rightarrow J / \psi(\psi(2 S), \ldots) K_{S}$. This is an important issue, if one wants to answer the question whether the weak angles measured in experiments that test $\mathrm{CP}$ violation match the angles that are being determined by measuring quantities that conserve $\mathrm{CP}$, e.g. the lenghts of the sides of the unitarity triangle 16.

The measurement is feasible: various amplitudes with different strong phases, corresponding to the decay chain $B^{0}\left(\overline{B^{0}}\right)(t) \rightarrow D^{+} D^{* *-}+$ $D^{-} D^{* *+}+\ldots \rightarrow D^{+} D^{-} \pi^{0}$, contribute to the final state producing sizeable interferences in the Dalitz plot. The coefficient of $\cos (2 \beta)$ in (7), $\operatorname{Im} \tilde{G}_{s}$, depicted in fig. , is expected to be different from zero over a large portion of the Dalitz plot, thus allowing the identification of the $\cos (2 \beta)$ term. The application of the method to other three-body modes has been investigated in ref. 115].
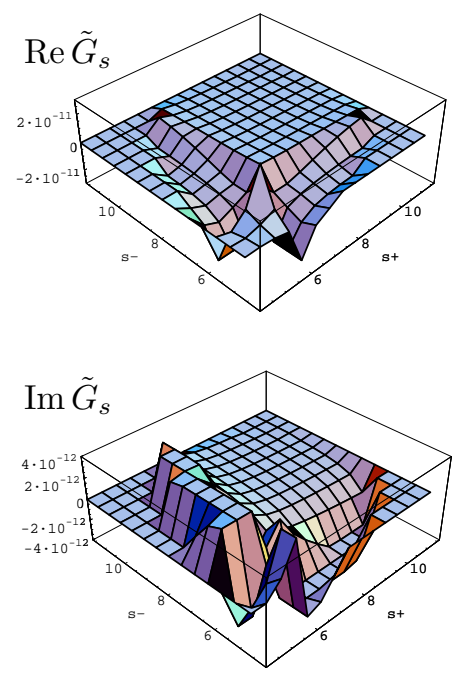

Figure 4. $\operatorname{Re} \tilde{G}_{s}\left(s_{+}, s_{-}\right)$(up) and $\operatorname{Im} \tilde{G}_{s}\left(s_{+}, s_{-}\right)$ (down) for the transition $B^{0} \rightarrow D^{+} D^{-} \pi^{0}$. Units of $s_{ \pm}=\left(p_{D^{ \pm}}+p_{\pi^{0}}\right)^{2}$ are $\mathrm{GeV}^{2}$.

\section{ACKNOWLEDGMENTS}

I thank F. De Fazio, G. Nardulli, N. Paver and Riazuddin for collaboration on the issues discussed in this talk.

\section{REFERENCES}

1. P. Colangelo and F. De Fazio, Phys. Lett. B 532 (2002) 193.

2. B. Aubert et al. [BABAR Collaboration], arXiv:hep-ex/0107056; arXiv:hep$\mathrm{ex} / 0207086$.

3. BELLE Collaboration, BELLE-CONF-0104.

4. See, e.g., M. Neubert and B. Stech, Adv. Ser. Direct. High Energy Phys. 15 (1998) 294.

5. M. B. Wise, Phys. Rev. D 45 (1992) 2188; G. Burdman and J. F. Donoghue, Phys. Lett. B 280 (1992) 287; T. M. Yan et al., Phys. Rev. D 46 (1992) 1148; A.F.Falk and M.Luke, Phys. Lett. B 292 (1992) 119.

6. P. Colangelo et al., Phys. Rev. D 52 (1995) 6422; P. Colangelo and F. De Fazio, Eur. Phys. J. C 4 (1998) 503.

7. See, e.g., F. De Fazio, arXiv:hep-ph/0010007.

8. D. Groom et al., Eur. Phys. J. C 15 (2000) 1.

9. P. Colangelo, G. Nardulli and M. Pietroni, Phys. Rev. D 43 (1991) 3002; P. Colangelo, F. De Fazio and G. Nardulli, Phys. Lett. B 334 (1994) 175.

10. A. Anastassov et al. [CLEO Collaboration], Phys. Rev. D 65 (2002) 032003.

11. S. Barlag et al. [ACCMOR Collaboration], Phys. Lett. B 278 (1992) 480.

12. For information on $g$ see, e.g., P. Colangelo and A. Khodjamirian, arXiv:hep-ph/0010175, A. Abada et al., arXiv:hep-ph/0206237, and references therein.

13. M. Beneke et al., Nucl. Phys. B 591 (2000) 313; C. W. Bauer, D. Pirjol and I. W. Stewart, Phys. Rev. Lett. 87 (2001) 201806.

14. Z. Luo and J. L. Rosner, Phys. Rev. D 64 (2001) 094001.

15. J. Charles et al., Phys. Lett. B 425 (1998) 375; P. Colangelo et al., Phys. Rev. D 60 (1999) 033002; T. E. Browder et al., Phys. Rev. D 61 (2000) 054009.

16. F. Parodi, these proceedings. 